\title{
Individual differences in visual attention: A short, reliable, open-source, and multilingual test of multiple object tracking in PsychoPy
}

\author{
Hauke S. Meyerhoff ${ }^{1} \cdot$ Frank Papenmeier $^{2}$ \\ Published online: 3 June 2020 \\ (C) The Psychonomic Society, Inc. 2020
}

\begin{abstract}
Individual differences in attentional abilities provide an interesting approach in studying visual attention as well as the relation of attention to other psychometric measures. However, recent research has demonstrated that many tasks from experimental research are not suitable for individual differences research, as they fail to capture these differences reliably. Here, we provide a test for individual differences in visual attention which relies on the multiple object tracking task (MOT). This test captures individual differences reliably in 6 to $15 \mathrm{~min}$. Within the task, the participants have to maintain a set of targets (among identical distractors) across an interval of object motion. It captures the efficiency of attentional deployment. Importantly, this test was explicitly designed and tested for reliability under conditions that match those of most laboratory research (restricted sample of students, approximately $n=50$ ). The test is free to use and runs fully under open-source software. In order to facilitate the application of the test, we have translated it into 16 common languages (Chinese, Danish, Dutch, English, Finnish, French, German, Italian, Japanese, Norwegian, Polish, Portuguese, Russian, Spanish, Swedish, and Turkish). The test can be downloaded at https://osf.io/qy6nb/. We hope that this MOT test supports researchers whose field of study requires capturing individual differences in visual attention reliably.
\end{abstract}

Keywords Multiple object tracking $\cdot$ Visual attention $\cdot$ Test $\cdot$ Individual differences $\cdot$ Attention

\section{Introduction}

Psychologists apply experimental as well as correlational techniques in order to test particular theories. Whereas both approaches typically focus on different levels of explanations, combined approaches studying variance between individuals as well as variance between experimental manipulations could be particularly powerful in order to understand, explain, and predict human cognition and behavior (Cronbach, 1957; see also Borsboom, Kievit, Cervone, \& Hood, 2009; Sternberg, \& Grigorenko, 2001). Such a unified perspective is typically not realized within the subdisciplines of psychology; however, there are some recent trends in cognitive psychology (a discipline typically focusing on the experimental approach)

Hauke S. Meyerhoff

h.meyerhoff@iwm-tuebingen.de

1 Leibniz-Institut für Wissensmedien, Schleichstr. 6, 72076 Tübingen, Germany

2 University of Tübingen, Tübingen, Germany acknowledging the fruitfulness of individual differences in evaluating theories on perceptual processes (Gauthier, 2018), attention (Huang, Mo, \& Li, 2012) and the formation of memory (Unsworth, 2019). A central challenge for combining correlational and experimental approaches arises from distinct considerations regarding reliabilities. Whereas experimental approaches generally aim to minimize variance between individual participants in order to obtain replicable effects of their manipulations, correlational approaches necessarily need substantial variance between observers in order to obtain stable rank orders of participants. The most intriguing consequence of these differences between the approaches is that paradigms which are known to produce reliable results in the experimental approach do not necessarily also produce reliable results in the correlational approach and vice versa. In contrast, several wellestablished paradigms from perception, attention, and cognitive research have revealed surprisingly low within-subject reliability scores.

This so-called reliability paradox has recently been demonstrated across several well-established research paradigms (Erikson Flanker Task, Stroop Task, Go/No-Go Task, StopSignal Task, Posner Cueing Task, SNARK Task, Navon 
Task) by Hedge, Powell and Sumner (2018). These authors measured the test-retest reliabilities of typical experimental variants of these tasks and observed unexpectedly low reliability measures given the widespread use of these tasks. The intra-class correlation (ICC) for different measures across these tasks varied from $0<\mathrm{ICC}<.82$ with most values ranging between $.50<\mathrm{ICC}<.75$. With such reliability scores true correlations of $r=.50$ would emerge as observable correlations around $.30<\mathrm{r}<.40$. As origin of this lack of reliability Hedge et al. have identified the restricted variance between different observers. This is plausible as variance between observers typically is noise in the experimental setups these tasks were designed for. Of course, this study does not rule out using tasks from experimental paradigms for studying individual differences, however, it shows that reliability cannot be taken for granted only because a particular paradigm has been used successfully in experimental research. Instead, adaptations might be necessary in order to introduce sufficient variance between individual observers.

In the current project, we focus on reliably capturing individual differences in visual attention. We present and make available an individual-differences version of a test of visual attention - an accuracy-based version of multiple object tracking (MOT) - that is a well-established experimental paradigm known to reliably capture effects of experimental manipulations. In order to facilitate unified research in the field of attention, we provide this test open access with instructions in a total of 16 languages $^{1}$ (Chinese, Danish, Dutch, English, Finnish, French, German, Italian, Japanese, Norwegian, Polish, Portuguese, Russian, Spanish, Swedish, and Turkish) and easy to analyze so that researchers both inside and outside the field of cognitive psychology can assess the corresponding individual capacity limitations of visual attention. Furthermore, we present data showing that this MOT test is highly reliable and thus also well suited for research investigating individual differences. We are aware of two related projects from other research laboratories. First, there is an open source code for conducting MOT experiments available at https://github.com/alexholcombe/ MOTcircular (see Holcombe \& Chen, 2012, 2013; Holcombe, Chen, \& Howe, 2014). Second, individual differences in MOT have also been focused on by Romeas, Guldner, and Faulbert (2016) as well as Tullo, Faulbert, and Bertone (2018). However, these studies focused on a commercial variant of MOT which is not openly available for research.

\section{Visual attention}

The extensive amount of visual information entering the visual system at any moment clearly exceeds the capacity

\footnotetext{
${ }^{1}$ We used a professional translation service for other languages than English and German. Please inform us if you spot errors in your language(s) or for the implementation of additional languages.
}

limitation of human attentional processing resulting in failures to detect relevant events in the field of view (e.g., Most, Scholl, Clifford, \& Simons, 2005; Simons \& Chabris, 1999; Rensink, O’Regan, \& Clark, 1997). In order to maintain function despite the overwhelming sensory stimulation, the cognitive system selects a subset of all available information for further processing. This selection mechanism is commonly referred to as attention. As early as 1890 , William James provided the most noted definition of attention:

"Everyone knows what attention is. It is the taking possession by the mind, in clear and vivid form, of one out of what seem several simultaneously possible objects or trains of thought. Focalization, concentration, of consciousness are of its essence. It implies withdrawal from some things in order to effectively deal with others, and is a condition which has a real opposite in the confused, dazed, scatterbrained state which in French is called distraction, and Zerstreutheit in German." (James, 1890, pp. 403-404)

Since then, human attention has been studied in thousands of reviewed articles and books and is probably one of the bestinvestigated psychological concepts. Nevertheless, defining visual attention has turned out to be (much) more complicated than the preceding quote suggests. Over the years, numerous research paradigms have been developed within the experimental approach that study different aspects of visual attention such as space-based processes (e.g., Jonides, 1981; Klein, 2000; Posner, 1980), focusing (e.g., Bahcall \& Kowler, 1999; Hopf et al., 2006; Müller et al., 2005), filtering (e.g., Eriksen \& Hoffman, 1972; Eriksen, 1995; Lavie, 1995), selection (e.g., McLeod, 1991; Raymond, Shapiro, \& Arnell, 1992; Stroop, 1935), the ability to search for a target among different objects (visual search, e.g., Treisman \& Gelade, 1980; Wolfe, 1994), as well as the ability to select multiple objects simultaneously (e.g., Franconeri, Alvarez, \& Enns, 2007; Pylyshyn \& Storm, 1988; Yantis, 1992).

In a seminal attempt to investigate the structure of visual attention, Huang et al. (2012) analyzed data from more than 250 participants performing a total of nine different attentional tasks (some participants up to 17 different tasks). From the shared variances in the paradigms under investigation, Huang et al. extracted a general factor of visual attention. On the one hand, several common attentional paradigms capturing the efficiency of visual attention such as visual search or tracking were substantially correlated with the general factor of visual attention. On the other hand, other common paradigms capturing biases in visual attention such as attentional capture and inhibition of return were remarkably dissociated from the general factor of visual attention (see also Pinto, van der Leij, Sligte, Lamme, \& Scholte, 2013). This pattern of results 
suggests that paradigms addressing the efficiency of visual attention indeed provide valid proxies for visual attention and thus are plausible candidates for tests reveling individual differences in attentional abilities between participants. Within this set of tested paradigms, MOT was among those which revealed the highest correlations with the general factor of visual attention as well as high split-half reliability estimates. Further, MOT has a clear-cut dependent variable (i.e., accuracy). Therefore, we considered MOT to be an ideal candidate for the development of an individual differences test in visual attention abilities for laboratory research.

\section{Multiple object tracking (MOT)}

\section{MOT as an experimental paradigm of visual attention}

Within the multiple object tracking task (originally developed by Pylyshyn \& Storm, 1988), participants track a subset of previously designated targets among indistinguishable objects over the duration of a trial (i.e., a few seconds). The capacity limitations of an individual observer under the current conditions can be estimated from the average of correctly identified targets after the interval of object motion (Hulleman, 2005). Three decades of experimental research have clearly demonstrated the deeply attentional nature of this task (for a review, see Meyerhoff, Papenmeier, \& Huff, 2017). Beyond the correlated individual differences with other attentional tasks there is further behavioral as well as neuroscientific evidence for the pronounced attentional demands of MOT. With regard to the behavioral evidence, Tombu and Seiffert (2008) demonstrated that MOT interferes with other attentional demanding dual tasks, but not with purely perceptual dual tasks. Indeed, the attentional demands of this task can become so exhaustive that tracking is capable of interfering with the extraction of the gist of a scene during natural scene perception, which typically only requires minimal attentional resources (Cohen, Alvarez, $\&$ Nakayama, 2011). With regard to neuroscientific evidence, Drew and colleagues (Drew \& Vogel, 2008; Drew, Horowitz, Wolfe, \& Vogel, 2011) demonstrated that the contralateral delay activity (CDA), which has been established as a measure of attentional load, is sensitive to the number of simultaneously tracked objects. Beyond these mostly attentional components, the MOT task probably also captures some aspects of attentional selection (Drew \& Vogel, 2008; Franconeri et al., 2007; Ma \& Flombaum, 2013) and working memory (e.g., Oksama \& Hyönä, 2004).

Importantly, previous research in the experimental approach has identified several parameters that allow for adjusting the difficulty of the MOT task (e.g., Alvarez \& Franconeri, 2007; Bettencourt \& Somers, 2009). With regard to the current project, this is important, as it enables us to set the task parameters so that they maximize variance between the different observers. For instance, MOT performance generally declines with an increasing number of to-be-tracked targets (e.g., Alvarez \& Franconeri, 2007; Drew et al., 2011; Pylyshyn \& Storm, 1988), an increasing number of distractors within the tracking area (e.g., Bettencourt \& Somers, 2009; Sears \& Pylyshyn, 2000), an increasing tracking duration (e.g., Oksama \& Hyönä, 2004), an increasing density of objects in the display (e.g., Bettencourt \& Somers, 2009; Franconeri, Jonathan, \& Scimeca, 2010), as well as an increasing object speed (e.g., Holcombe \& Chen, 2012; Meyerhoff, Papenmeier, Jahn, \& Huff, 2016; Tombu \& Seiffert, 2011). Please note that this list represents exemplary manipulations only and makes no claim to be complete.

\section{Considerations for the development of an individual differences MOT test}

The central aim of this project was to establish a MOT test that reliably captures individual differences in tracking capacity. Although individual-difference estimates derived from experimental tasks from research on visual attention - including MOT - are capable of delivering reliable estimates of individual differences (Huang et al., 2012), the work of Hedge et al. (2018) clearly demonstrates that this cannot be taken for granted but needs to be established empirically. Therefore, we not only provide the test itself but also report a demonstration of its reliability (for a demonstration of the validity of MOT as an attentional task, see Huang et al., 2012). Our attempt to establish the individual differences test for MOT capacity was based on the following five considerations.

First, our test is supposed to support research on individual differences (i.e., it is not designed for clinical assessment). With this consideration in mind, we applied task parameters that our previous research (e.g., Meyerhoff et al., 2016) has identified as optimal for inducing effects of experimental manipulations as well as allowing a maximum of individual differences. In other words, the task parameters such as the number of tracked objects and their speed were set to values that an average sample of students (as used in most psychology studies) reveals a mean performance in the middle between guessing rate and ceiling.

Second, based on the first consideration, our MOT test needs to also deliver reliable estimates of individual differences within sample sizes of approximately 50 participants because the necessity of much larger sample sizes would be impractical for most research projects. As limits in the reliability of a particular test also limit the potential correlations between this test and other psychometric measures (Nunnally, 1970; Spearman, 1904), the reliability needs to be $r>.80$ in order allow for detecting correlations with other psychometric measures having reasonable sample sizes.

Third, the test should be relatively short in order to fit into regular research protocols. Our main goal was for the task 
itself not to exceed 15 min (i.e., much shorter than a typical experimental study relying on this paradigm). For research protocols with more intense time pressure, we also aimed to establish even shorter versions of this task (which come at the risk of reduced reliability).

Fourth, the test should be open source and free to run on most of the common computer systems without the necessity to buy additional software or licenses. Further, the usage of this test should be easy so that no coding abilities are necessary to conduct independent research with the task. Of course, this also applies to the analysis of the outcome of the test, which needs to be provided in a manner that is suitable for open-source tools of data analysis.

Fifth and finally, we aimed to provide the test, including instructions, in many different languages in order to facilitate its distribution across different countries and cultures. We hope that this encourages researchers across the world to use established measures of individual differences rather than developing their own paradigms because an inflation of testing procedures decreases the comparability among the different research projects.

\section{Open-source MOT test in PsychoPy}

In this section, we will describe the MOT test and provide the necessary information to run the test and analyze the data. We first provide the full method details of the task that are necessary to describe the test in scientific reports. We then provide the technical requirements and preparations that are necessary to run the MOT test. In most cases, these preparations need to be done only once on each computer. Finally, we provide instructions on how to run the test with participants and how to analyze the obtained data.

\section{Description of the task}

This task follows the standard procedure of accuracy-based multiple object tracking (see Fig. 1). Each trial begins with the presentation of an empty gray square frame (20 x $20 \mathrm{deg})$ appearing against a black background. After $500 \mathrm{~ms}$, eight white discs with a diameter of $1.3^{\circ}$ of visual angle are positioned inside the frame. Four of them are designated as targets by flashing on and off in red, three times in intervals of $200 \mathrm{~ms}$ and finally remaining red for $400 \mathrm{~ms}$ before they turn back to white. Next, all objects start to move on randomly selected straight paths for $8 \mathrm{~s}$ at a constant object speed of $8^{\circ}$ of visual angle per second (deg/s). When touching the surrounding frame, the objects are reflected with the angle of incidence equaling the angle of reflection. Following the interval of object motion, all objects remain stationary at their latest location and the mouse pointer appears onscreen. The participants then use the mouse to mark all targets that they were able to track and guess the remaining ones. When four of the discs have been marked, the participants receive feedback regarding the number of correctly identified targets in this trial (e.g., "3 out of 4 correct"). The next trial is initiated by pressing the space bar. There are two distinct sets of trials if retesting should be necessary ${ }^{2}$ (in standard cases, we recommend using the same set for each participant). Within each set all participants complete the same trials in the same order. The rationale behind this is to avoid unwanted variance arising from differences in trial order in order to maximize the proportion of variance arising from individual differences. The test is organized into five blocks consisting of ten trials each. Before the first block of the test, the participants complete five practice trials of which the results are not analyzed. Completing the full task requires approximately $15 \mathrm{~min}$.

\section{Technical prerequisites and preparations for running the test}

The MOT test is scripted using the open source libraries of PsychoPy (Peirce, 2007). Therefore, it can be run on almost all Linux, Macintosh, and Windows machines. The test requires a monitor running at $60 \mathrm{~Hz}$, which can display at least $30 \mathrm{x}$ $22 \mathrm{deg}$ of visual angle at a reasonable viewing distance ${ }^{3}$. The central prerequisite is to download and install PsychoPy3 from https:/github.com/psychopy/psychopy/ releases/tag/3.2.4. We recommend the standalone version of PsychoPy3 (3.2.4). When using the MOT test for the first time on a particular computer system (this includes the screen) it is necessary to adjust the monitor settings appropriately. To do this, open the monitor center in PsychoPy (in the tools setting) and enter screen resolution, viewing distance, as well as the monitor dimensions. It is important that this monitor setting is labeled MOTtestMonitor (if labeled differently, the test will not run, but return an according error message). Finally, download our MOT test from https://osf.io/qy6nb/ and unzip it into a separate folder on the system.

\section{Running the MOT test}

In order to run the MOT test, open PsychoPy3 and switch to the Coder view (i.e., not the Builder view, which is the initial default setting) using the "view" settings. Next, open the file MOTtest.py from inside the PsychoPy coder and press the (green) run button. The first window will ask you to select your language. This window, the descriptive variables (to match with the logfile), and error messages are in English, whereas any

\footnotetext{
${ }^{2}$ If researchers prefer to determine the reliability of this test with a test-retest procedure themselves, the distinct sets of trials could be run in two sessions with the same participant ID.

${ }^{3}$ With current releases of PsychoPy3 (i.e., at least until PsychoPy 2020.1.3, which was the latest version available and tested while writing this manuscript) the MOT test cannot be run correctly on retina displays. Users of these machines can run the code on attached standard external monitors.
} 


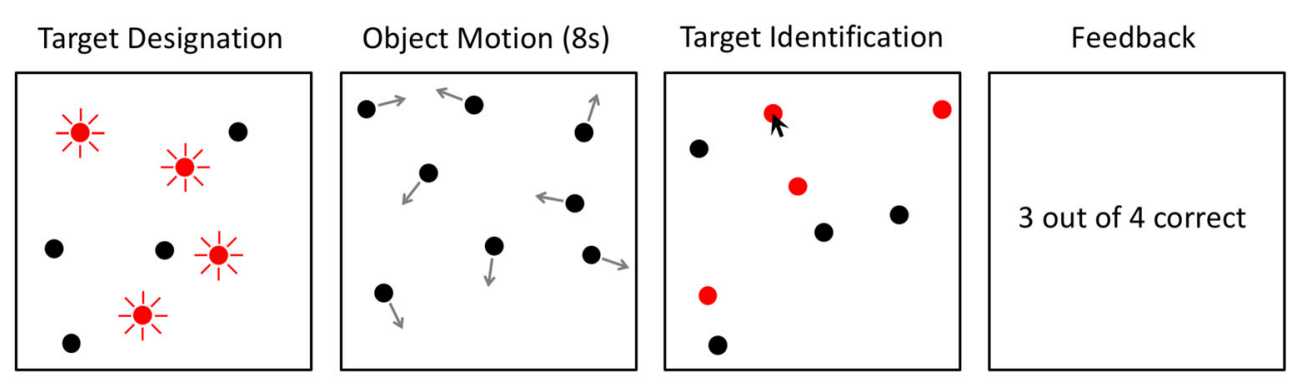

Time

Fig. 1 Illustration of the multiple object tracking task (black/white inverted). Four out of eight objects are designated as targets by flashing in red. After that, the objects are indistinguishable and move for eight

other window, task instructions, and feedback are in the selected language. The second window asks for the subject ID, the set of motion paths ( 1 or 2 depending on which sets of motion paths you want to use), as well as a description of the participating subject, which is typically reported in vision experiments (i.e., age, gender, handedness, vision impairments). We strongly recommend using the same set of trials for all participants of one sample (we have used both sets for estimating the reliability). Further, we recommend not to test participants that do not have normal or corrected-to-normal vision.

Following the descriptive variables, the test switches into a full screen mode and the participants see a task instruction onscreen (in the selected language). This instruction first includes information about the duration of the task. Next, the participants are instructed that eight discs will appear at the beginning of each trial. The instruction states that the participants are supposed to track the discs flashing in red across an interval of object motion and select them at the end of the trial using the mouse pointer (which is invisible during the trial).

Once the MOT test has entered the full-screen mode, it can be stopped only by typing in the word 'quit' whenever a text screen is shown. After completion of the full test, the final screen asks the participants to call the experimenter.

\section{Data collection and analysis}

Our MOT test supports three different modes of data collection and analysis. The first and fastest way of accessing the individual data is to press the key " $\mathrm{d}$ " in the final screen of the experiment. If used, this option immediately shows the average of correctly identified objects as well as the corresponding tracking capacity (i.e., corrected for guessing) based on Formula 1 as published in Hulleman (2005).

$m=n(2 p-1)$

In this formula, $m$ indicates the tracking capacity (i.e., corrected for guessing), $n$ indicates the number of targets seconds. Following the motion interval, the participants select the four objects that they expect to be the targets and receive feedback

and $p$ indicates the proportion of correctly identified targets. Please note that this option is not explicitly mentioned on the last screen to avoid that participants who are not supposed to receive feedback on their performance become aware of this possibility.

In order to avoid a manual transfer of the tracking capacity for further analysis, the second option is a short logfile which is saved into the log-folder upon completion of the task (i.e., there is no such logfile when the test is canceled manually). This short logfile contains only the identifier of the participant (labelled 'participantID'), the average of correctly identified targets per trial ('avTargetsCorrect') and the accompanying tracking capacity ('capacity').

Finally, there is a full logfile reporting performance on a trial-by-trial basis. This logfile is necessary to analyze information that goes beyond pure tracking capacity (i.e., such as performance over time, etc.). This logfile is in the long format (i.e., it contains one line for each trial of the task). This logfile includes the identifier of the participant ('subjectID'), descriptive statistics from the initial window of the test ('set', 'nBlocksTotal', 'age', 'sex', 'handiness', 'visionImpairment'), information about the block and trial number ('block', 'trial'), the number objects ('nObjects'; always eight in this test), the number of targets (nTargets; always four in this test), one column for each presented object, indicating whether it was selected as target at the end of the trial ('o1marked', 'o2marked', ..., 'o8marked'), as well as the sum of correctly identified targets ('sumCorrect'; the targets were always the four first objects in the logfile). Independent of the preferred output file, we recommend emptying the log folder before collecting data from a new sample and to regularly backup the logfiles to avoid a potential loss of data.

Beyond immediate analyses of tracking capacity, the exact motion patterns of the individual trials might be of interest for some research projects. For these projects, every screen location and motion direction are available in the trial-files within the "motionPaths_60Hz" folder. Each line in these files refers to one frame of the motion paths. There are four columns for each object (i.e., the fifth column is the first column of the 
second object). The first column refers to the $x$-location on screen (in degrees of visual angle). The second column refers to the $y$-location on screen (in degrees of visual angle). The third column refers to the motion direction which results from these locations $(0-360$, with 0 being rightward motion, 90 being motion to the top, etc.). The fourth column refers to the motion speed, which is a constant of $8^{\circ}$ of visual angle per second.

\section{Reliability of the provided test}

Previous research has demonstrated that tasks from experimental research do not necessarily provide stable individual differences (Hedge et al., 2018). In order to demonstrate the suitability of the MOT test for research on individual differences, we conducted a study measuring the reliability of the MOT test. For this, we used a test-retest procedure with the two different sets of motion paths. As most laboratory psychology research is run with student samples (i.e., a rather restricted sample with a sample size of approximately $n=$ 50), we also used a student sample to demonstrate the suitability of the MOT test for exactly such a scenario.

Importantly, the actual reliability depends on the exclusion criteria for poorly performing participants. Some researchers have suggested that chance level in MOT should be defined as a tracking capacity of one object (e.g., Hulleman, 2005). In our task, however, the objects can overlap with each other, so that confusion might occur even when tracking only one object. Thus, we think that the exclusion of participants with a tracking capacity below one object (i.e., $\mathrm{m}<1$ ) might be too conservative as an exclusion criterion whereas not excluding any participants might be too liberal. We think that a scenario in which participants are not able to track at least one object every second trial is a reasonable exclusion criterion (i.e., $\mathrm{m}<$ $0.5)$. Certainly, participants who fail to track any objects beyond guessing should be excluded from the sample (i.e., $\mathrm{m}<$ 0.1 ). To allow individual researchers to set an exclusion criterion that fits their demands, we report reliability estimates for all four discussed exclusion criteria.

\section{Methods}

\section{Participants}

Fifty students from the University of Tübingen participated in the test (39 female, 11 male, 19-31 years) in return for monetary compensation or course credit. All participants reported normal or corrected-to-normal vision. The experimental procedure was approved by the internal review board of the Leibniz-Institut für Wissensmedien, Tübingen, and all participants provided informed consent prior to testing. The data of the last four trials in the second session were lost for one subject due to an unexpected program termination (i.e., data of this subject for the last block of both sessions included only six trials).

\section{Apparatus, stimuli, and procedure}

All stimuli were presented on a 23-inch LCD screen $(60 \mathrm{~Hz}$, 1920 x 1200 pixels) controlled by a MacMini at an unrestricted viewing distance of approximately $60 \mathrm{~cm}$. Please note the description of the task section for a full description of the stimuli and procedure of the MOT test. All participants completed both sets of trials of the test with a break of approximately 15 min between both sessions. Each set of trials took approximately $15 \mathrm{~min}$ to complete (including instructions). The instructions prior to the experiment as well as any information during the tasks were presented in German.

\section{Results}

In order to assess the reliability of the MOT test, we will report correlations between test and retest as well as intraclass correlations. Both measures indicate a high reliability of the MOT test. Because tracking capacity provides a more straightforward interpretation than the sum of correctly identified targets, we first transformed the number of correctly identified objects into tracking capacity values using the Formula 1.

\section{Full test}

We analyzed the reliability of the full test by correlating the capacity estimates of each participant between the two test sessions using Pearson's product-moment correlation. Capacity estimates prior to any exclusions are available in Fig. 2. To determine whether a participant should be excluded from the reliability estimate, we calculated the average capacity across both runs of the test for each participant. For the recommended exclusion criterion of $\mathrm{m}<0.5$ (i.e., two excluded participants), we observed a strong correlation $r(46)=.87$, $p<.001,95 \% \mathrm{CI}[.78, .93]$ indicating a very high reliability of the multiple object tracking task. To facilitate comparability to previous related research (e.g., Hedge et al., 2018), we additionally calculated the Intraclass Correlation Coefficients (ICC) using the ICC libraries in R (Wolak \& Wolak, 2015). Again, we observed a remarkably high reliability estimate $\mathrm{ICC}=.86,95 \% \mathrm{CI}[.76, .92]$. Reliability estimates for other exclusion criteria are summarized in Table 1.

\section{Partial test}

In order to test whether using shorter versions of the multiple object tracking task also provide substantial reliabilities, we repeated the reliability analysis with differently large subsets of blocks of the test (see Fig. 3). As for the reliability estimate 


\section{Full Test}

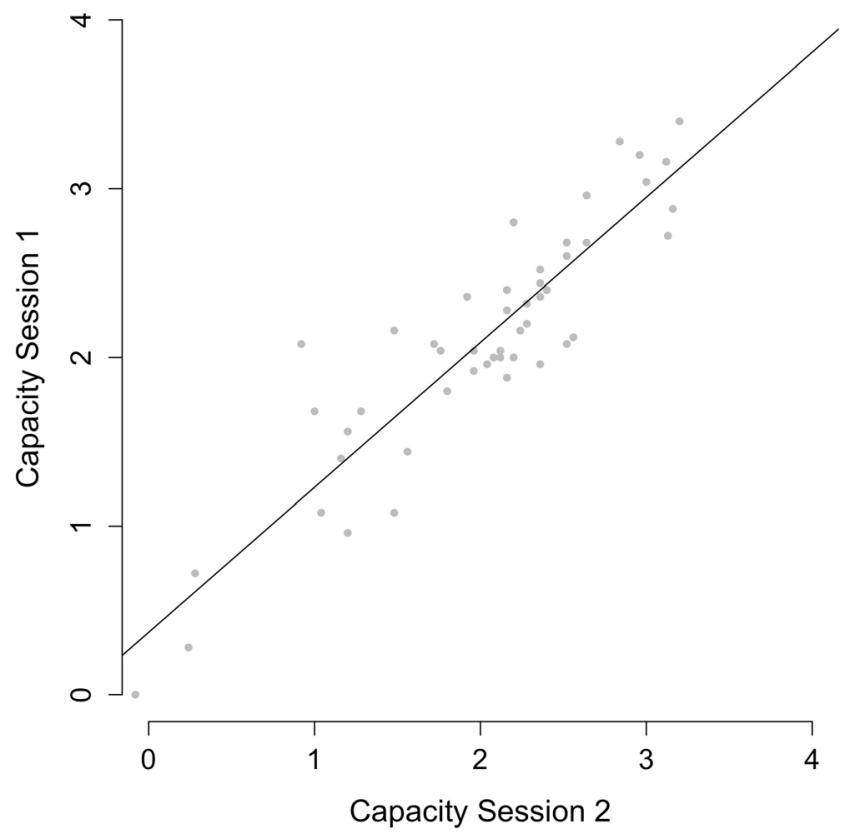

Fig. 2 Capacity estimations for the full test (five blocks of trials) prior to any exclusions. Each dot indicates observed capacity of an individual participant in both sessions. The reliability of the test varies with different exclusion criteria (see main text for details)

for the full test, we applied our recommended exclusion criterion of $\mathrm{m}<0.5$ separately to each subset of our data (reliability estimates for other exclusion criteria are available in Table 1). When only analyzing the first four blocks (i.e., $80 \%$ of the full test) the reliability estimates were $r(46)=.84, p<.001,95 \%$ CI $[.72, .91]$, ICC $=.82,95 \%$ CI $[.70, .89]$. When analyzing only the first three blocks (i.e., $60 \%$ of the full test), the reliability estimates were $r(46)=.78, p<.001,95 \%$ CI $[.64$, $.87], \mathrm{ICC}=.78,95 \% \mathrm{CI}[.63, .87]$. When analyzing only the first two blocks (i.e., $40 \%$ of the full test), the reliability estimates were $r(46)=.76, p<.001,95 \% \mathrm{CI}[.61, .86], \mathrm{ICC}=.76$, $95 \%$ CI $[.61, .86]$. Finally, when analyzing only the very first block (i.e., $20 \%$ of the full test), the reliability estimates were $r(47)=.66, p<.001,95 \%$ CI $[.46, .79]$, ICC $=.64,95 \% \mathrm{CI}$ $[.44, .78]$.

\section{General discussion}

The central aim of this project was to distill a variant of the MOT task that is suitable to reliably identify individual differences among observers. Given the recent observation from Hedge et al. (2018) that many laboratory tasks from experimental research lack in sufficient variance between observers to capture individual differences reliably, this aim was pressing to allow for combined experimental and correlational research in the field of attention. With regard to this aim, we were able to show the capability of our task to reliably identify individual differences in the reported study. Indeed, the observed reliabilities were very high across all possible exclusion criteria. In particular, the full version of the task was in the range of an excellent diagnostic test irrespective of the applied exclusion criterion. Nevertheless, even the shorter versions down to two blocks of trials (approx. $6 \mathrm{~min}$ ) still revealed decent reliabilities in the range of a good diagnostic test irrespective of the applied exclusion criterion. As we were exploring a standard variant of MOT which has previously been used in

Table 1 Reliability of the MOT test for different exclusion criteria

\begin{tabular}{|c|c|c|c|c|c|c|}
\hline & \multicolumn{6}{|c|}{ Exclusion criterion } \\
\hline & \multicolumn{3}{|c|}{ No exclusions } & \multicolumn{3}{|c|}{$\mathrm{m}<0.1$} \\
\hline & $n$ & $r[95 \% \mathrm{CI}]$ & $\mathrm{ICC}[95 \% \mathrm{CI}]$ & $n$ & $r[95 \% \mathrm{CI}]$ & $\mathrm{ICC}[95 \% \mathrm{CI}]$ \\
\hline Full test & 50 & $.91[.84, .95]$ & $.90[.83, .94]$ & 49 & $.89[.81,95]$ & $.88[.80, .93]$ \\
\hline $80 \%$ Test & 50 & $.87[.78, .93]$ & $.86[.77, .92]$ & 49 & $.86[.76, .92]$ & $.84[.73, .91]$ \\
\hline $60 \%$ Test & 50 & $.83[.72, .90]$ & $.83[.72, .90]$ & 49 & $.81[.68, .89]$ & $.80[.68, .88]$ \\
\hline $40 \%$ Test & 50 & $.81[.69, .89]$ & $.82[.70, .89]$ & 49 & $.78[.64, .87]$ & $.79[.65, .87]$ \\
\hline \multirow[t]{4}{*}{$20 \%$ Test } & 50 & $.69[.51, .81]$ & $.68[.50, .81]$ & 49 & $.66[.46, .79]$ & $.64[.44, .78]$ \\
\hline & \multicolumn{6}{|c|}{ Exclusion criterion } \\
\hline & \multicolumn{3}{|c|}{$\mathrm{m}<0.5$ (recommended) } & \multicolumn{3}{|c|}{$\mathrm{m}<1$} \\
\hline & $n$ & $r[95 \% \mathrm{CI}]$ & $\operatorname{ICC}[95 \% \mathrm{CI}]$ & $n$ & $r[95 \% \mathrm{CI}]$ & $\mathrm{ICC}[95 \% \mathrm{CI}]$ \\
\hline Full test & 48 & $.87[.78, .93]$ & $.86[.76, .92]$ & 47 & $.85[.74, .91]$ & $.84[.73, .91]$ \\
\hline $80 \%$ Test & 48 & $.84[.72, .91]$ & $.82[.70, .89]$ & 45 & $.79[.65, .88]$ & $.77[.62, .87]$ \\
\hline $60 \%$ Test & 48 & $.78[.64, .87]$ & $.78[.63, .87]$ & 46 & $.74[.58, .85]$ & $.74[.58, .85]$ \\
\hline $40 \%$ Test & 48 & $.76[.61, .86]$ & $.76[.61, .86]$ & 45 & $.72[.54, .83]$ & $.73[.55, .84]$ \\
\hline $20 \%$ Test & 49 & $.66[.46, .79]$ & $.64[.44, .78]$ & 43 & $.60[.36, .76]$ & $.60[.36, .76]$ \\
\hline
\end{tabular}

$m$ tracking capacity, $n$ remaining sample size, $r$ Pearson correlation, $I C C$ intraclass correlation coefficient 
$80 \%$ Test

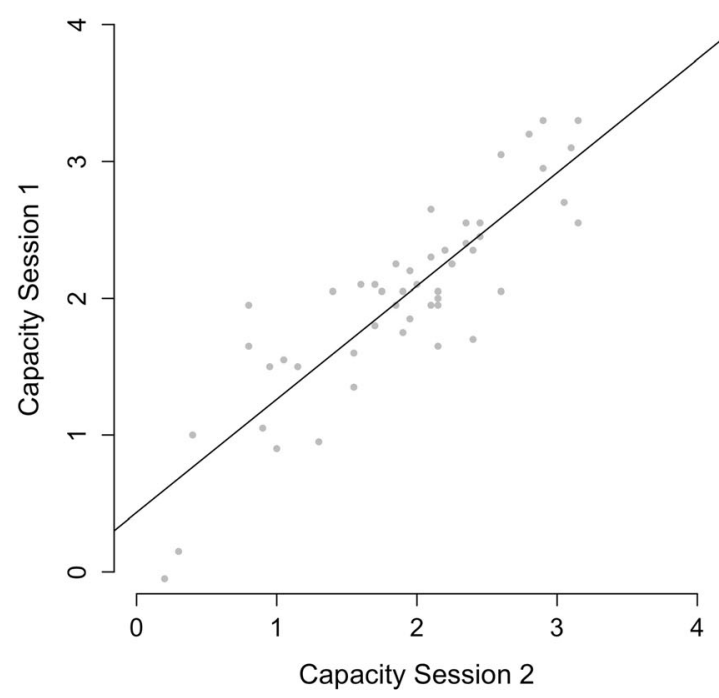

$40 \%$ Test

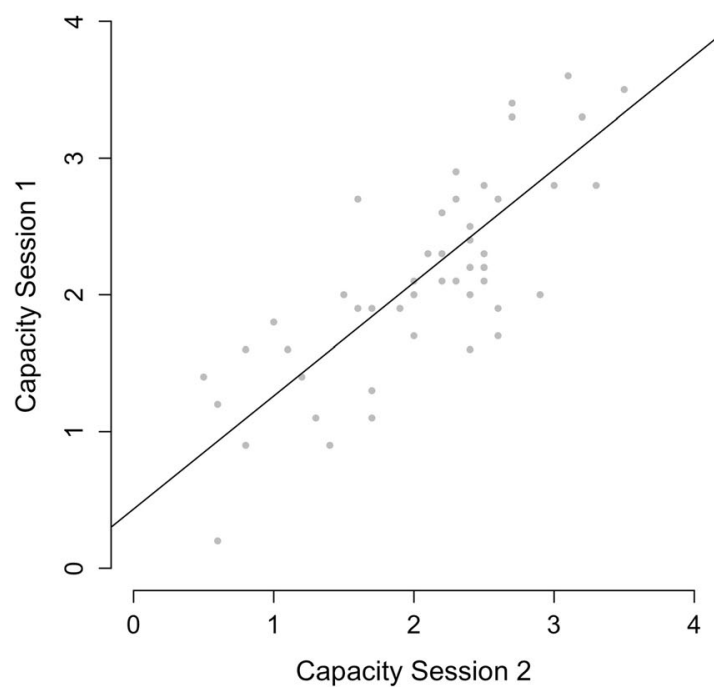

Fig. 3 Capacity estimation of the partial testing prior to any exclusions. For this estimation, we only analyzed trials from blocks 1-4 (upper left panel), blocks 1-3 (upper right panel), blocks 1 and 2 (lower left panel),

experimental research (e.g., Meyerhoff et al., 2016) this finding shows that standard MOT still shows sufficient variation between observers allowing to capture individual differences at high reliability scores. With regard to this variance, standard MOT seems to differ from the standard versions of the experimental paradigms that were tested for their reliability in individual differences research in the study of Hedge et al. (2018).

As the validity of MOT as a measure of attentional processes has also been demonstrated previously (Cohen et al., 2011; Huang et al., 2012; Tombu \& Seiffert, 2008), our data
$60 \%$ Test

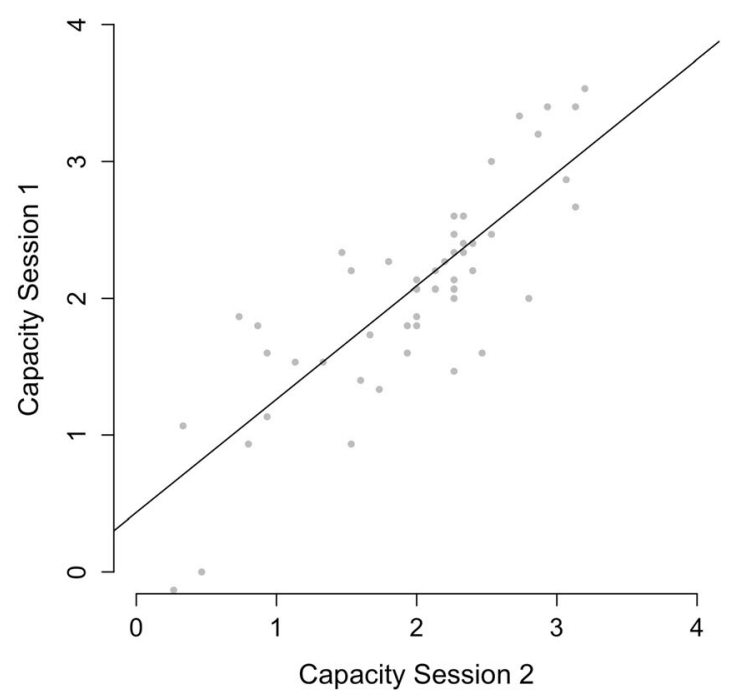

$20 \%$ Test

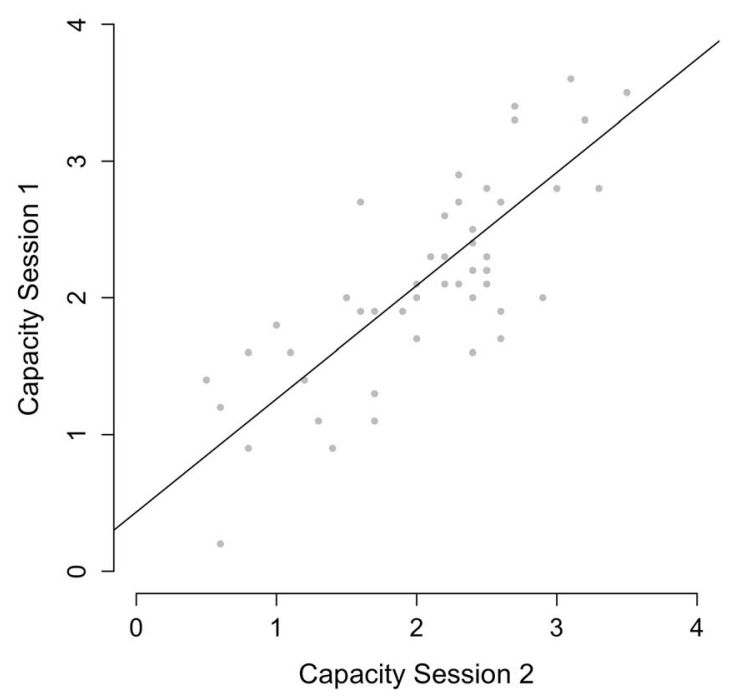

or only block 1 (lower right panel). Each dot indicates observed capacity of an individual participant in both sessions. The reliability of the test varies with different exclusion criteria (see main text for details)

suggests that the MOT task reflects a reliable measure of individual differences in attentional capability. A standard caveat that applies to all research on individual differences with performance measures (i.e., to our task too), however, is that individual differences in the motivation to perform the task might contribute to the high reliability scores. Nevertheless, there is one aspect in our test that is capable of reducing the impact of motivation on the reliability estimate. Immediately after marking the tracked objects, the participants received feedback about their performance. In a related, but (yet unpublished) experiment, we observed that 
providing feedback substantially increases the motivation to perform the MOT task.

\section{Combining experimental and correlational research}

As in all fields of general psychology, experimental research is dominant in research on visual attention as it allows for drawing causal inferences. However, research on individual differences could also provide a valuable contribution to the formation of theories on visual attention. For instance, the systematic analysis of individual differences in visual attention by Huang et al. (2012) has revealed that attentional efficiency (such as MOT) and attentional bias (such as cueing effects; Posner, 1980; Jonides, 1981) are rather unrelated and thus most likely reflect independent components of visual attention (see also Pinto et al., 2013). Indeed, combining data from correlational and experimental research appears to be a promising approach to forwarding attentional theories; however, conducting correlational research with tasks and paradigms from experimental research is difficult as these paradigms are mostly designed to minimize individual differences allowing more room for effects of experimental manipulations (and vice versa). Here, our MOT task might contribute an interesting piece to the overall puzzle as it seems to allow for correlational as well as experimental research. With regard to individual differences, the high reliability estimates indicate the suitability of the MOT task for this type of research. At the same time, this task with identical parameters has already been used successfully in experimental research (see the control condition in Meyerhoff et al., 2016). Therefore, this particular task might enable researchers to combine the causal inferences from experimental research with the generalizations from correlational research in order to provide stronger conclusions than from one of the approaches alone.

\section{Scope and limitations}

An important thing to consider when using an individual differences test is the scope and the accompanying limitations of the test at hand. In our case, the MOT test was explicitly designed for the typical lab setup based on an average student sample. Therefore, all task parameters are set in a manner that generates the most variance between individual observers in this sample or a comparable one. As any substantial deviation from this distribution would lower the reliability of the test, our MOT test in its current state is therefore not suitable for investigating samples that deviate substantially from the performance of students such as young children (Brockhoff et al., 2016; O'Hearn, Landau, \& Hoffman, 2005) or very old participants (Störmer, Li, Heekeren, \& Lindenberger, 2011). If these samples are of interest, the test needs to be adapted to take the expected reduced overall performance into consideration. This could encompass lowering tracking load, lowering object speeds, lowering object density or other factors that are related to the general difficulty of the MOT task (Meyerhoff et al., 2017). Further, this version of the task is not suitable for clinical diagnostics as it does not come along with normative data and most probably has an insufficient resolution of individual differences in the lower ranges of ability. To appropriately address these types of questions, the test would need to measure more precisely in the lower ranges; however, such trials would not contribute much in distinguishing between individuals in the normal ranges. Therefore, we did not include such trials in the present version of the test as this would unnecessarily prolong the testing duration for its main purpose, namely, identifying individual difference in student samples.

\section{Conclusions}

Research on individual differences provides a powerful approach to testing and developing psychological theories. Here, we provide a test for visual attention (translated in 16 languages) that captures reliable individual differences in a typical lab-based setting (i.e., a rather homogeneous student sample of approximately $n=50$ ). We hope and think that this test could be a useful tool in studying the relation between individual differences in attention and other psychometric measures, as well as promoting combined research on individual differences and effects of experimental manipulations.

Acknowledgements All materials, scripts, and data of this project are available at https://osf.io/ws58q/. We would like to thank Anne Müller for her help with the data collection.

Open Science Statement The data and materials for all experiments are available at the Open Science Framework (https://osf.io/ws58q/).

\section{References}

Alvarez, G. A., \& Franconeri, S. L. (2007). How many objects can you track?: Evidence for a resource-limited attentive tracking mechanism. Journal of Vision, 7(13):14, 1-10.

Bahcall, D. O., \& Kowler, E. (1999). Attentional interference at small spatial separations. Vision Research, 39, 71-86.

Bettencourt, K. C., \& Somers, D. C. (2009). Effects of target enhancement and distractor suppression on multiple object tracking capacity. Journal of Vision, 9(7):9, 1-11.

Borsboom, D., Kievit, R. A., Cervone, D., \& Hood, S. B. (2009). The two disciplines of scientific psychology, or: The disunity of psychology as a working hypothesis. In J. Valsiner, P. C. M. Molenaar, M. C. D. P. Lyra, \& N. Chaudhary (Eds): Dynamic Process Methodology in the Social and Developmental Sciences (pp. 67-97). Springer, New York, NY.

Brockhoff, A., Papenmeier, F., Wolf, K., Pfeiffer, T., Jahn, G., \& Huff, M. (2016). Viewpoint matters: Exploring the involvement of reference frames in multiple object tracking from a developmental perspective. Cognitive Development, 37, 1-8. 
Cohen, M. A., Alvarez, G. A., \& Nakayama, K. (2011). Natural-scene perception requires attention. Psychological Science.

Cronbach, L. J. (1957). The two disciplines of scientific psychology. American Psychologist, 12, 671-684.

Drew, T., \& Vogel, E. K. (2008). Neural measures of individual differences in selecting and tracking multiple moving objects. The Journal of Neuroscience, 28, 4183-4191.

Drew, T., Horowitz, T. S., Wolfe, J. M., \& Vogel, E. K. (2011). Delineating the neural signatures of tracking spatial position and working memory during attentive tracking. The Journal of Neuroscience, 31, 659-668.

Eriksen, C. W. (1995). The flanker task and response competition: A useful tool for investigating a variety of cognitive problems. Visual Cognition, 2, 101-118.

Erisken, C. W., \& Hoffman, J. E. (1972). Temporal and spatial characteristics of selective encoding from visual displays. Perception \& Psychophysics, 12, 201-204.

Franconeri, S. L., Alvarez, G. A., \& Enns, J. T. (2007). How many locations can you select? Journal of Experimental Psychology: Human Perception and Performance, 33, 1003-1012.

Franconeri, S. L., Jonathan, S. V., \& Scimeca, J. M. (2010). Tracking multiple objects is limited only by object spacing, not speed, time, or capacity. Psychological Science, 21, 920-925.

Gauthier, I. (2018). Domain-specific and domain-general individual differences in visual object recognition. Current Directions in Psychological Science, 27, 97-102.

Hedge, C., Powell, G., \& Sumner, P. (2018). The reliability paradox: Why robust cognitive tasks do not produce reliable individual differences. Behavior Research Methods, 50, 1166-1186.

Holcombe, A. O., \& Chen, W. Y. (2012). Exhausting attentional tracking resources with a single fast-moving object. Cognition, 123, 218228.

Holcombe, A. O., \& Chen, W. Y. (2013). Splitting attention reduces temporal resolution from $7 \mathrm{~Hz}$ for tracking one object to $<3 \mathrm{~Hz}$ when tracking three. Journal of Vision, 13(1):12, 1-19.

Holcombe, A. O., Chen, W. Y., \& Howe, P. D. (2014). Object tracking: Absence of long-range spatial interference supports resource theories. Journal of Vision, 14(6):1, 1-21.

Hopf, J.-M., Boehler, C. N., Luck, S. J., Tsotsos, J. K., Heinze, H.-J., \& Schoenfeld, M. A. (2006). Direct neurophysiological evidence for spatial suppression surrounding the focus of attention in vision. Proceedings of the National Academy of Sciences, 103, 1053-1058.

Huang, L., Mo, L., \& Li, Y. (2012). Measuring the interrelations among multiple paradigms of visual attention: an individual differences approach. Journal of Experimental Psychology: Human Perception and Performance, 38, 414-428.

Hulleman, J. (2005). The mathematics of multiple object tracking: From proportions correct to number of objects tracked. Vision Research, 45, 2298-2309.

James, W. (1890). The principles of psychology (Vol. 1). New York, NY: Holt.

Jonides, J. (1981). Voluntary versus automatic control over the mind's eye. In: J. Long \& A. Baddeley (Eds.), Attention and performance $I X$. Hillsdale, N.J.: Lawrence Erlbaum Associates.

Klein, R. M. (2000). Inhibition of return. Trends in Cognitive Sciences, 4, $138-147$

Lavie, N. (1995). Perceptual load as a necessary condition for selective attention. Journal of Experimental Psychology: Human Perception and Performance, 21, 451-468.

Ma, Z., \& Flombaum, J. I. (2013). Off to a bad start: Uncertainty about the number of targets at the onset of multiple object tracking. Journal of Experimental Psychology: Human Perception and Performance, 39, 1421-1432.

McLeod, C. M. (1991). Half a century of research on the Stroop effect: An integrative review. Psychological Bulletin, 109, 163-203.
Meyerhoff, H. S., Papenmeier, F., Jahn, G., \& Huff, M. (2016). Not FLEXible enough: Exploring the temporal dynamics of attentional reallocations with the multiple object tracking paradigm. Journal of Experimental Psychology: Human Perception and Performance, 42, 776-787.

Meyerhoff, H.S., Papenmeier, F., \& Huff, M. (2017). Studying visual attention using the multiple object tracking paradigm: A tutorial review. Attention, Perception, \& Psychophysics, 79, 1255-1274.

Most, S. B., Scholl, B. J., Clifford, E. R., \& Simons, D. J. (2005). What you see is what you set: Sustained inattentional blindness and the capture of attention. Psychological Review, 112, 217-242.

Müller, N. G., Mollenhauer, M., Rösler, A., \& Kleinschmidt, A. (2005). The attentional field has a Mexican hat distribution. Vision Research, 45, 1129-1137.

Nunnally, J. C. (1970). Introduction to psychological measurement. New York, NY, USA: McGraw-Hill.

O'Hearn, K., Landau, B., \& Hoffman, J. E. (2005). Multiple object tracking in people with Williams syndrome and in normally developing children. Psychological Science, 16, 905-912.

Oksama, L., \& Hyönä, J. (2004). Is multiple object tracking carried out automatically by an early vision mechanism independent of higherorder cognition? An individual difference approach. Visual Cognition, 11, 631-671.

Peirce, J. W. (2007). PsychoPy-psychophysics software in Python. Journal of Neuroscience Methods, 162, 8-13.

Pinto, Y., van der Leij, A. R., Sligte, I. G., Lamme, V. A. F., \& Scholte, H. S. (2013). Bottom-up and top-down attention are independent. Journal of Vision, 13(3):16, 1-14.

Posner, M. I. (1980). Orienting of attention. The Quarterly Journal of Experimental Psychology, 32, 3-25.

Pylyshyn, Z. W., \& Storm, R. W. (1988). Tracking multiple independent targets: Evidence for a parallel tracking mechanism. Spatial Vision, 3, 179-197.

Raymond, J. E., Shapiro, K. L., \& Arnell, K. M. (1992). Temporary suppression of visual processing in an RSVP task: An attentional blink? Journal of Experimental Psychology: Human Perception and Performance, 18, 849-860.

Rensink, R. A., O'Regan, J. K., \& Clark, J. J. (1997). To see or not to see: The need for attention to perceive changes in scenes. Psychological Science, 8, 368-373

Romeas, T., Guldner, A., \& Faubert, J. (2016). 3D-Multiple Object Tracking training task improves passing decision-making accuracy in soccer players. Psychology of Sport and Exercise, 22, 1-9.

Sears, C. R., \& Pylyshyn, Z. W. (2000). Multiple object tracking and attentional processing. Canadian Journal of Experimental Psychology, 54, 1-14.

Simons, D. J., \& Chabris, C. F. (1999). Gorillas in our midst: Sustained inattentional blindness for dynamic events. Perception, 28, 10591074.

Spearman, C. (1904). The proof and measurement of association between two things. American Journal of Psychology, 15, 72-101.

Sternberg, R. J., \& Grigorenko, E. L. (2001). Unified psychology. American Psychologist, 56, 1069-1079.

Störmer, V. S., Li, S.-C., Heekeren, H. R., \& Lindenberger, U. (2011) Feature-based interference from unattended visual field during attentional tracking in younger and older adults. Journal of Vision, 11, $1-12$.

Stroop, J. R. (1935). Studies of interference in serial verbal reactions. Journal of Experimental Psychology, 18, 643-662.

Tombu, M., \& Seiffert, A. E. (2008). Attentional costs in multiple-object tracking. Cognition, 108, 1-25.

Tombu, M., \& Seiffert, A. E. (2011). Tracking planets and moons: Mechanisms of object tracking revealed with a new paradigm. Attention, Perception, \& Psychophysics, 73, 738-750.

Treisman, A., \& Gelade, G. (1980). A feature integration theory of attention. Cognitive Psychology, 12, 97-136. 
Tullo, D., Faubert, J., \& Bertone, A. (2018). The characterization of attention resource capacity and its relationship with fluid reasoning intelligence: A multiple object tracking study. Intelligence, 69, 158168.

Unsworth, N. (2019). Individual differences in long-term memory. Psychological Bulletin, 145, 79-139.

Wolak, M., \& Wolak, M. M. (2015). R-Package 'ICC'. Facilitating estimation of the Intraclass Correlation Coefficient.
Wolfe, J. M. (1994). Guided Search 2.0: A revised model of visual search. Psychonomic Bulletin \& Review, 1, 202-238.

Yantis, S. (1992). Multielement visual tracking: Attention and perceptual organization. Cognitive Psychology, 24, 295-340. doi:https://doi. org/10.1016/0010-0285(92)90010-Y

Publisher's note Springer Nature remains neutral with regard to jurisdictional claims in published maps and institutional affiliations. 